\title{
A NOTE ON A WEIGHTED SOBOLEV INEQUALITY
}

\author{
FILIPPO CHIARENZA AND MICHELE FRASCA ${ }^{1}$
}

\begin{abstract}
We give a simple proof of a weighted imebedding theorem whose proof was originally given in $[\mathbf{3}]$.
\end{abstract}

The purpose of this note is to provide a simplified proof of a weighted imbedding theorem previously proved by Fabes, Kenig and Serapioni [3]. They proved the following inequality (see Theorem (1.2) in $[\mathbf{3}]$ ),

$$
\left(\frac{1}{w\left(B_{R}\right)} \int_{B_{R}}|u(x)|^{k p} w(x) d x\right)^{1 / k p} \leq c R\left(\frac{1}{w\left(B_{R}\right)} \int_{B_{R}}|\nabla u|^{p} w(x) d x\right)^{1 / p} .
$$

Here $w \in A_{p}(1<p<+\infty), 1 \leq k \leq n /(n-1)+\delta$ and $u$ is any function in $C_{0}^{\infty}\left(B_{R}\right)$ (henceforth $w\left(B_{R}\right)=\int_{B_{R}} w d x$ and $n$ is the dimension).

Our short proof, inspired by an idea in $[\mathbf{4}]$, is an easy consequence of the boundedness of the Hardy-Littlewood maximal operator $M f$ between weighted $L^{p}$ spaces iff the weight is $A_{p}$ (see $[\mathbf{5}, \mathbf{2}]$ and, for an elementary proof, the recent paper $[\mathbf{1}]$ ). In the following assume the reader is familiar with the relevant definitions and notations as given in $[\mathbf{3}]$.

Set $I f(x)=\int_{R^{n}}|f(y)||x-y|^{1-n} d y$. It is well known that (1) is an immediate consequence of the following inequality:

(2) $\left(\frac{1}{w\left(B_{R}\right)} \int_{B_{R}}[I f(x)]^{k p} w(x) d x\right)^{1 / k p} \leq c R\left(\frac{1}{w\left(B_{R}\right)} \int_{B_{R}}|f(x)|^{p} w(x) d x\right)^{1 / p}$.

Here $w \in A_{p}(1<p<+\infty), 1 \leq k \leq n /(n-1)+\delta, f \in L^{p}\left(B_{R}, w\right)$, and $c, \delta$ are positive constants independent on $f$ and $R$.

To prove (2) set, for any $\varepsilon>0$,

$$
I^{(\varepsilon)} f(x)=\int_{|x-y| \leq \varepsilon}|f(y)||x-y|^{1-n} d y .
$$

It is easy to see that $I^{(\varepsilon)} f(x) \leq c \varepsilon M f(x)$. Further,

$$
I f(x)-I^{(\varepsilon)} f(x) \equiv I_{(\varepsilon)} f(x)
$$

$$
\begin{array}{r}
\leq\|f\|_{L^{p}\left(B_{R}, w\right)}\left(\int_{\{|x-y|>\varepsilon\} \cap B_{R}}|x-y|^{(1-n) p^{\prime}} w^{-1 /(p-1)} d y\right)^{1 / p^{\prime}}, \\
\left(\frac{1}{p}+\frac{1}{p^{\prime}}=1\right) .
\end{array}
$$

Received by the editors April 5, 1984.

1980 Mathematics Subject Classification. Primary 46E35; Secondary 42B25.

Key words and phrases. Weighted inequalities, Sobolev spaces, maximal functions.

${ }^{1}$ This work was partially financially supported by a national project of the Italian Ministero della Pubblica Istruzione and by G.N.A.F.A. Consiglio Nazionale delle Ricerche. 
Because $w \in A_{p}$, a $q$ can be chosen such that $w \in A_{q}, 1<q<p, n-p / q>0$ (see $[2])$. Hence,

$$
I_{(\varepsilon)} f(x) \leq c\|f\|_{L^{p}\left(B_{R}, w\right)}\left(\int_{B_{R}}[w(y)]^{-1 /(q-1)} d y\right)^{(q-1) / p} \varepsilon^{1-n q / p} .
$$

(3) and (4) imply

$$
\text { (5) } \quad I f(x) \leq c \varepsilon M f(x)+c\|f\|_{L^{p}\left(B_{R}, w\right)}\left(\int_{B_{R}}[w(y)]^{-1 /(q-1)} d y\right)^{(q-1) / p} \varepsilon^{1-n q / p} \text {. }
$$

We minimize with respect to $\varepsilon$ the right side of (5) to get

$$
I f(x) \leq c[M f(x)]^{1-p / n q}\|f\|_{L^{p}\left(B_{R}, w\right)}^{p / n q}\left(\int_{B_{R}}[w(y)]^{-1 /(q-1)} d y\right)^{(q-1) / n q}
$$

and, using the boundedness of $M f$ in the $L^{p}\left(B_{R}, w\right)$ norm,

$$
\|I f(x)\|_{L^{p k}\left(B_{R}, w\right)} \leq c\|f\|_{L^{p}\left(B_{R}, w\right)}\left(\int_{B_{R}}[w(y)]^{-1 /(q-1)} d y\right)^{(q-1) / n q},
$$

where $k=n q /(n q-p)$. To complete the proof we divide by $\left[w\left(B_{R}\right)\right]^{(n q-p) / n p q}$ and use the $A_{q}$ condition.

\section{REFERENCES}

1. M. Christ and R. Fefferman, A note on weighted norm inequalities for the Hardy-Littlewood maximal operator, Proc. Amer. Math. Soc. 87 (1983), 447-448.

2. R. Coifman and C. Fefferman, Weighted norm inequalities for maximal functions and singular integrals, Studia Math. 51 (1974), 241-250.

3. E. Fabes, C. Kenig and R. Serapioni, The local regularity of solutions of degenerate elliptic equations, Comm. Partial Differential Equations 7 (1982), 77-116.

4. L. Hedberg, On certain convolution inequalities, Proc. Amer. Math. Soc. 36 (1972), 505-510.

5. B. Muckenhoupt, Weighted norm inequalities for the Hardy maximal function, Trans. Amer. Math. Soc. 165 (1972), 207-226.

Seminario Matematico, Università di Catania, Viale A. Doria, 6, I-95125 CataNIA, ITALY 